NASA Contractor Report 3206

\title{
A Simplified Model for the Viscous Crossflow in a Slotted Test Section
}

Charlie H. Cooke

GRANT NSG-1517

NOVEMBER 1979

\section{NASA}


NASA Contractor Report 3206

\section{A Simplified Model for the Viscous Crossflow in a Slotted Test Section}

Charlie H. Cooke

Old Dominion University Research Foundation

Norfolk, Virginia

Prepared for

Langley Research Center

under Grant NSG-1517

\section{N/SN}

National Aeronautics

and Space Administration

Scientific and Technical Information Branch 
NUMERICAL ANALYSIS . . . . . . . . . . . . . . . . . . . . . . 3

The Flow Domain. . . . . . . . . . . . . . . . . . . . 3

Numerical Algorithm . . . . . . . . . . . . . . . . 3

COMPUTATIONAL RESULTS . . . . . . . . . . . . . . . . . . . . . . . 7

SIMILARITY TO EXPERIMENT $\quad$. . . . . . . . . . . . . . . . . . . . . . . 10

DISCUSSION AND CONCLUSIONS . . . . . . . . . . . . . . . . . . . 10

REFERENCES . . . . . . . . . . . . . . . . . . . . . . . . . . 12

\section{LIST OF FIGURES}

Figure

$\underline{\text { Page }}$

1 (a) Computational domain and boundary conditions . . . . . . . . . 15

1(b) Coordinate system (alternate lines parallel to wall omitted) $6 \times 1$ magnified, horizontal x-direction........... 16

2 Slot flow profiles: viscous vs. inviscid.......... . . 17

3 Body pressure distribution; $\mathrm{Re}=100$, openness ratio $=0.1 . \cdot 19$

4 Body pressure distribution; $\operatorname{Re}=1000$, openness ratio $=0.1 .20$

5 Slot normal velocity component at slat center: viscous $(\operatorname{Re}=100)$ vs. inviscid flow . . . . . . . . . . . . . . 21

6 Experimental pressure distribution through slot under two conditions of flow . . . . . . . . . . . . . . . . . 22 

SUMMARY

A simplified physical model is constructed which simulates the viscous crossflow in a fluid layer near the slots at a fixed streamwise location in a slotted wind tunnel. For low to moderate Reynolds numbers, numerical solutions of the two-dimensional, incompressible Navier-Stokes equations in stream function and vorticity, which govern the model flow, are obtained. Fairly general slot geometry is incorporated by means of the Thompson-Thames-Mastin transformation. An approximate factorization scheme with cyclic acceleration parameters is employed to solve a finite difference analog of the stream function equation. The vorticity equation is numerically solved with a modified version of the classical alternating direction implicit (ADI) scheme. Although no quantitative asscssment of solution accuracy can be made, numerical results for variations in incremental wall pressure around the slat are at least qualitatively similar to some experimental results of Berndt and Sorenson [21]. 


\section{INTRODUCTION}

Longitudinal slots are often employed in wind-tunnel test sections to reduce adverse wall effects. However, the capability to correct wind-tunnel data in order to account for boundary-inúced flow distortions is still rather limited. Analyses resulting in formulations of the slotted wall boundary condition [1-6] are usually based upon inviscid representations of the flow. On the other hand, it is conjectured by some that viscous effects of an unknown and complicated nature are present; therefore, results from the inviscid models become suspect [7]. The inviscid theory has been rather well developed and some experimental verification of flow models has been attempted [1-11]. Nevertheless, alleged inconsistencies in trying to app $1 y$ theoretical results in practical flows often result in placing reliance upon empirical methods in the design and use of slotted test sections. 
The purpose of the present investigation is to provide some insight into the effects of viscosity on slot flows. The approach employed is a viscous crossflow analysis in a transverse plane. Suppose it is assumed than an aerofoil extends the breadth of the tunne1. By reasoning analogous to the slender body theory, it is argued classically $[2-6,11]$ that the inviscid perturbation crossflow at a fixed longitudinal station in a test section with multiple slots above and/or below the model can be approximated in a fluid layer near the slots by solution of the two-dimensional velocity potential equation. The present treatment is a viscous generalization of such flow models, through use of the two-dimensional, time-dependent, incompressible Navier-Stokes equations and numerical solution techniques.

This approach does not satisfactorily model the flow, particularly since streamwise (down-tunnel) boundary-layer growth and interaction is not accounted for. However, some insight into Reynolds number effects and flow separation in the vicinity of the slot should be provided. Due to the complicated geometry involved, ordinary finite difference numerical methods are expected to encounter difficulty with treatment of boundary conditions unless coupled with transformation techniques. The Thompson-Thames-Mastin (TTM) transformation [12] is an aid in attaining more accurate results for general shapes. By this method the geometric restrictions which are inherent in classical flow models due to idealizations in slat shape, e.g., infinitely thin slats and slots with sidewalls (infinite depth), can be avoided. 


\section{NUMERICAL ANALYSIS}

The Flow Domain

The computational domain and a cross-sectional view of the slot geometry for the two-dimensional problem are provided by figure 1 . Flow symmetry with respect to centers of slot and slat is assumed. The problem is driven by slat-generated vorticity and by a prescribed inflow at the top of the computational domain, on the tunnel side of the flow. We remark that prescribing the inflow also prescribes mass flow rate at outflow, although the strict form of velocity distribution at the outflow can be altered from what it is at inflow. Boundary conditions for the case of identically prescribed mass flux distributions at inflow and outflow are provided by figure $1(a)$.

\section{Numerical Algorithm}

In order to simplify the numerical solution procedure, the domain of figure 1 (a) is mapped onto a rectangular region by means of the TTM transformation. A typical finite difference mesh generated for the problem using the Thompson grid-generation package is given by figure 1(b). Taking the transformed coordinates to be $x$ and $y$, the twodimensiona1, time-dependent, incompressible Navier-Stokes equations in dimensionless stream function-vorticity form for the transformed problem are now given:

$$
\frac{\partial \zeta}{\partial t}+u \frac{\partial \zeta}{\partial x}+v \frac{\partial \zeta}{\partial y}=\frac{1}{J^{2} R}\left[\alpha \frac{\partial^{2} \zeta}{\partial x^{2}}-2 \beta \frac{\partial^{2} \zeta}{\partial x \partial y}+\gamma \frac{\partial^{2} \zeta}{\partial y^{2}}\right]
$$




$$
\frac{\sigma \psi_{y}+\tau \psi_{x}+\alpha \psi_{x x}-2 \beta \psi_{x y}+\gamma \psi_{y y}}{J^{2}}=-\zeta
$$

Here the functions of $x, y$, denoted by $\alpha, \beta, \gamma, \sigma, \tau, J$, are transformation coefficients; $R$ is the Reynolds number; $\zeta$ is vorticity; $\psi$ is stream function; and the quantities $u, v$ are related to transformed velocity components. Exact expressions for $u, v$ and the transformation coefficients are given in reference 13.

The problem now is the numerical solution of equations (1) and (2) on a rectangular region. Whatever algorithm is applied to equation (1), the overall efficiency is determined by the manner in which equation (2) is solved, which solution can occupy as much as 90 percent of the total time required [14]. Classically, this difficulty has been alleviated through use of fast Poisson solvers applied to equation (2). Such methods are inapplicable in the case of variable transformation coefficients. Therefore, in the present study the method chosen for solving equation (2) is an approximate factorization scheme [15] which amounts to the use of the alternating direction implicit technique (ADI) with cyclic, accelerated convergence parameters [16]. This method has proved superior to the popularly used successive line overrelaxation techniques for the equations of transonic potential flow [15].

Let

$$
C_{n}=\psi_{n+1}-\psi_{n}
$$

be the correction at stage $\mathrm{n}$ to an estimate $\psi_{\mathrm{n}}$ of the stream function at a lattice point, and let $R_{n}$ be the residual when equation (2) is 
approximated using second order, centered difference derivative approximations, and $\psi_{\mathrm{n}}$ is substituted. The stream function iteration is given by

$$
\left(S-\alpha \delta_{x x}\right)\left(S-\gamma \delta_{y y}\right) C_{n}=S w R_{n}
$$

Here $\delta_{x x}, \delta_{y y}$ are second order, centered difference operators and equation (4) results in tridiagonal systems of 1 inear equations to be solved in the customary alternating direction fashion. With $\omega=2$, the parameter $S$ is cyclically varied to speed convergence by reducing error-frequency components in a band determined by the maximum and minimum magnitudes of the transformation coefficients $\alpha, \gamma$. At the interior nodes, the values of the quantities $u, v$ and the transformation coefficients $\alpha, \beta, \gamma, \sigma, \tau, J$ are obtained by centered differencing of the expressions given for these variables in reference 13.

Once having obtained the velocity-related quantities $u$ and $v$ from a stream function solution of equation (2), the vorticity can be updated by solving equation (1). For efficiency, the time step employed should be the maximal restricted only by requirements of accuracy, and the unconditional linear stability of the classical ADI schemes lends itself to satisfaction of this criterion. We have therefore chosen to solve equation (1) using Briley's implementation [14] for the ADI scheme of Douglas [17].

The ADI scheme is applied by splitting the time step into two equal parts. Over the first half-step the following equation approximates equation (1): 


$$
\begin{aligned}
& \frac{\zeta_{i, j}^{n+1 / 2}-\zeta_{i, j}^{n}}{\frac{1}{2} \Delta t}+u_{i, j}^{*}\left[\frac{\zeta_{i+1, j}^{n}-\zeta_{i-1, j}^{n}}{2 \Delta x}\right] \\
& +v_{i, j}^{*}\left[\frac{\zeta_{i, j+1}^{n+1 / 2}-\zeta_{i, j-1}^{n+1 / 2}}{2 \Delta y}\right]=\frac{1}{J^{2} R}\left[\alpha_{i, j}\left(\frac{\zeta_{i+1, j}^{n}-2 \zeta_{i, j}^{n}+\zeta_{i-1, j}^{n}}{\Delta x^{2}}\right)\right. \\
& +\gamma_{i, j}\left(\frac{\left.\zeta_{i, j+1}^{n+1 / 2}-2 \zeta_{i, j}^{n+1 / 2}-\frac{\zeta_{i, j}^{n+1 / 2}}{\Delta y^{2}}\right)-2 \beta_{i, j} \frac{\partial^{2} \zeta^{*}}{\partial x \partial y}}{2}\right]
\end{aligned}
$$

The equation for the second half-step is

$$
\begin{aligned}
& \frac{\zeta_{i, j}^{n+1}-\zeta_{i, j}^{n+1 / 2}}{\frac{1}{2} \Delta t}+u_{i, j}^{*}\left[\frac{\zeta_{i+1, j}^{n+1}-\zeta_{i-1, j}^{n+1}}{2 \Delta x}\right] \\
& +v_{i, j}^{*}\left[\frac{\zeta_{i, j+1}^{n+1 / 2}-\zeta_{i, j-1}^{n+1 / 2}}{2 \Delta y}\right]=\frac{1}{J^{2} R}\left[\alpha_{i, j}\left(\frac{\zeta_{i+1, j}^{n+1}-2 \zeta_{i, j}^{n+1}+\zeta_{i-1, j}^{n+1}}{(\Delta x)^{2}}\right)\right. \\
& +\gamma_{i, j}
\end{aligned}
$$

The approximations $(5 a, b)$ become second order accurate in space and time when $u_{i, j}^{*}, v_{i, j}^{*}$ and the mixed derivatives $\bar{\zeta}_{x y}^{*}$ are evaluated at time $t_{n+1 / 2}$. This is accomplished by half-step predictions of the form

$$
f_{n+1 / 2}=\frac{3}{2} f_{n}-\frac{1}{2} f_{n-1}+o(\Delta t)^{2}
$$

Second order accurate space differencing is applied to $\zeta_{\mathrm{xy}}{ }^{\text {. }}$ 
For equations $(5 a, b)$, boundary values of vorticity are prescribed zero except along the wall (which is transformed to the right boundary of the computational plane). Here a vorticity distribution at the new step is computed using the full step prediction equation (7).

$$
\zeta_{n+1}=2 \zeta_{n}-\zeta_{n-1}+0(\Delta t)^{2}
$$

Upon solving equations $(5 a, b)$ and updating the stream function through equation (4), a corrected vorticity distribution is calculated. This process is repeated several times, to converge wall vorticity prior to proceeding to the next step. On the first correction the new vorticity distribution at the wall is obtained by using second order accurate backward differences in equation (2) wherever necessary. Thereafter the Israeli correction [18]

$$
\zeta_{\text {wa11 }}^{\mathrm{m}+1}=\zeta_{\text {wa11 }}^{\mathrm{m}}+\left.\mathrm{k} \frac{\sqrt{\alpha}}{\mathrm{J}} \quad \frac{\partial \psi}{\partial \mathrm{x}}\right|_{\text {wal1 }}
$$

is applied to iteratively induce the no-slip condition. Here the derivative $\frac{\partial \psi}{\partial x}$ at the wall is evaluated third order accurate using backward differences, since near separation points convergence of the iteration was found to be sensitive to this parameter. The parameter $\mathrm{K}$ is constant for all wall points, but is increased in magnitude as tangential velocity decreases. The proper choice of the sign of $K$ that will induce no-slip is discussed in reference 19 .

\section{COMPUTATIONAL RESULTS}

In this section preliminary numerical solutions are presented for viscous slot flow at Reynolds numbers of 100 and 1000 , with a slot thick- 
ness ratio and slot openness ratio (slot length to slot spacing) of 0.1 . Openness and thickness ratios are realistic; however the fully rounded slot shape of figure 1 is not known to have been used by wind tunnel designers. Customarily, slat shapes have been nearly rectangular or trapezoidal, with small radii of curvature (as low as 1/160 of the slat length) produced by rounding sharp corners.

Figure 2 exhibits comparisons between viscous and inviscid slot flow stream function and vorticity profiles. Figures 3 and 4 show pressure distribution around the slat, relative to plenum-side stagnation pressure. The quantity plotted is

$$
\Lambda P(y)=P(y)-P_{s}=\int_{y_{s}}^{y} \frac{1}{R J}\left(\alpha \zeta_{x}-\beta \zeta_{y}\right) d y
$$

where $y$ is the transformed coordinate which varies along the wall and $P_{S}$ is the plenum pressure at the center of the slat. The y-plot scale has been normalized to vary from zero to one around the slat, with 0.5 the coordinate of slot center. Figure 5 shows the character of the boundary layer across the center of the slot for the $\mathrm{Re}=100$ normal component of velocity. Values of $-v$ are plotted. The inviscid normal velocity distribution across the slot is also shown.

These results were obtained using a 51 by 51 grid, which is uniform in the transformed plane, but highly irregular in the physical plane, with order of magnitude variations in step size [see fig. 1(b)]. We remark that a boundary-layer packing function has been applied so as to concentrate the majority of points near the wall and in the vicinity of the slot: The scale of figure 2 is approximately actual size, or $1.0 \times$ 
8.2. The calculations were stopped when the maximum change in wall vorticity became the order of $10^{-4}$, with a maximum wall velocity value of magnitude $10^{-4}$.

Approximately 700 time steps using $\Delta t=0.0001$ were required to converge the $\mathrm{Re}=1000$ calculation, with starting flow field initialized using the $\mathrm{Re}=100$ solution. The code required $153 \mathrm{k}_{8}$ core storage locations, of which approximately $31 \mathrm{k}_{8}$ were used to store metric coefficients and transformation-related quantities. Typical Cyber 175 average CPU time for the advancing one time step is $2.2 \times 10^{-4}$ seconds per step per computational node per model equation. of course, to advance each time step requires a number of iterations to converge wall vorticity in order to yield an acceptably small level of wall velocity, and each vorticity iteration requires a few iterations (an average of four after impulsive start effects damp out) to converge the stream function field. No real comparisons of efficiency between algorithms can be made unless the same problem is solved by another method; however, it appears that Rubin's modification [20] to the strongly implicit method of Stone could perhaps produce more efficient convergence at the expense of some additional core storage.

Flow calculations at $\operatorname{Re}=5000$ becamc unstable. This is probably due to insufficient mesh refinement. It is expected that, as the Reynolds number increases, thinning of the boundary layer near the lip of the slot in the vicinity of minimum wall pressure can quickly lead to problems with mesh refinement. It is not known at which point turbulent transition brought on by increasing Reynolds number might produce physical instability. 


\section{SIMILARITY TO EXPERIMENT}

Consider the calculated slat pressure distributions of figures 3 and 4. As the flow enters the slot, a distinct pressure drop resulting from geometric flow acceleration is observed. A slight recompression precedes flow separation. For the case $\mathrm{Re}=100$, a pressure plateau occurs, followed by a gradual pressure recovery on the plenum side of the slat. However, the inference implied by the pressure data for $\mathrm{Re}=$ 1000 is that, as the Reynolds number increases, viscous effects due to the separation bubble produce yet another pressure drop prior to the final recovery stage.

It is expected that this second drop becomes increasingly sharp at higher Reynolds numbers, continuing the trend observed from figures 3 and 4. Some support for this inference may be gleaned from experimental data of Berndt and Sorenson [21], which is reproduced in figure 6 .

Although slat shapes, slat thickness and openness ratios, and Reynolds numbers are somewhat dissimilar, comparison of figures 4 and 5 (with pressure axis positive-direction reversed for figure 5) shows qualitative agreement for pressure data measured close to the aerofoil (position $\mathrm{x}=-22.5)$.

\section{DISCUSSION AND CONCLUSIONS}

In any numerical study the accuracy of results is subject to question, particularly as in cases such as this where theoretical results are not available for comparison. Grid refinement has not been attempted, although our feeling drawn from previous numerical experiences is that the grid is a little coarse in the vicinity of the slot, and possibly near 
plenum stagnation. However, the qualitative similarity of numerical and experimental wall pressure distributions is certainly encouraging. Furthermore, the results of figures 2 and 3 appear qualitatively reasonable. The flow separates in a region of adverse pressure gradient; the pressure then temporarily flattens in the separation region as the separation point (body distance equal to 0.54 ) is passed. The separation point is past the lip of the slot on the plenum side, and the circulation bubble is clearly defined. The flow does not appear to reattach; this may be due to numerical error, perhaps caused by the wall vorticity iteration. We conclude that overall the numerical algorithm has performed well, although its efficiency is hindered by the wall vorticity calculation. The approximate factorization algorithm for the stream function calculation appears remarkably efficient, requiring very few iterations to convergence once the calculation damps out the impulsive start effects.

Finally, we emphasize that this report represents only a cursory study of the viscous slot flow problem. Further efforts appear necessary, with much that can be done using the present code in terms of investigating the effects on the flow of slot geometry and Reynolds number. Moreover, three-dimensional models which incorporate the effects of turbulence and streamwise boundary layers are necessary for meaningful insight into the slot flow mechanism. 


\section{REFERENCES}

1. Sune B. Berndt and H. Sorenson, Flow properties of slotted walls for transonic test sections. No. 17, AGARD F1uid Dynamics Pane1 Symposium on Wind Tunnel Design and Testing Techniques, London, Oct. 6-8, 1975.

2. Don D. Davis and Dewey Moore, Analytical study of blockageand lift-interference corrections for slotted tunnels obtained by the substitution of an equivalent homogeneous boundary for the discrete slots. NACA RM L53E07b, Washington, DC, June 1953.

3. G. Guderly, Simplifications of the boundary conditions at a wind tunnel wall with longitudinal slots. WADC TR 53-150, Jan. 11,1954 .

4. Pau1 F. Maeder and Albert D. Wood, Transonic wind tunnel test sections. J. Appl. Math. and Phys. 7, 177-212 (1956).

5. C.F. Chen and J.W. Mears, Experimenta1 and theoretical study of mean boundary conditions at perforated and longitudinally slotted wind tunnel walls. AEDC TR-57-20, Dec. 1957.

6. Richard W. Barnwel1, Improvements in the slotted wall boundary condition. Proceedings, AIAA 9th Aerodynamic Testing Conference, Arlington, TX, June 7-9, 1976.

7. Sune B. Berndt, Inviscid theory of wall interference in slotted test sections. AIAA, 15, 1278-1287 (Sept. 1977).

8. Richard W. Barnwel1 and Joel L. Everhart, A parametric experimental study of the interference effects and the 
boundary condition coefficient of slotted wind tunnels. Proceedings, AIAA 10th Aerodynamic Testing Conference, San Diego, CA, Apr. 19-21, 1978.

9. Richard W. Barnwe11, Design and performance evaluation of slotted walls for two-dimensional wind tunnels. NASA TM 78618, Feb. 1978 .

10. P. Lofgren, Simplifications of boundary condition at a slotted wind tunnel wall with boundary layer. AD-A010 504, European Office of Aerospace Research and Development, London, Mar. 1975.

11. W.W. Wood, Tunnel interference from slotted walls. Quart. J. Mech. Appl. Math. 12, 126-140 (May 1964).

12. Joe J. Thompson, Frank C. Thames, and C. Wayne Mastin, Boundary fitted curvilinear coordinate systems for solution of partial differential equations on fields containing any number of arbitrary two dimensional bodies. NASA CR-2729, July 1977.

13. Frank C. Thames, Joe F. Thompson, and C. Wayne Mastin, Numerical solution of the time dependent Navier-Stokes equations about arbitrary two-dimensional bodies. J. Comp. Phys. 24, 245-273 (July 1977).

14. W.R. Briley, A numerical study of laminar separation bubbles using the Navier-Stokes equations. J. Fluid Mech. 47, $713-736(1971)$

15. T.L. Holst, An implicit algorithm for the conservative transonic full potential equation using an arbitrary 
mesh. No. 78-113, AIAA 11th Fluid and Plasma Dynamics Conference, Seatt1e, WA, Ju1y 10-12, 1978.

16. Richard S. Varga, Matrix Iterative Analysis, Prentice-Ha11, Englewood Cliffs, $\mathrm{NJ}, 1963$.

17. Patrick J. Roache, Computational Fluid Dynamics, Hermosa Publishers, Albuquerque, NM, 1972.

18. M. Israeli, A fast implicit numerical method for time dependent viscous flows. Studies in Applied Mathematics, Vo1. XLIX, No. 4, Dec. 1970.

19. C.H. Cooke, On artificial vorticity in numerical simulation of incompressible flow. Proceedings, Annual Spring Meeting, SIAM, Toronto, Canada, June 10-13, 1979.

20. S.G. Rubin and P.K. Khosla, Navier-Stokes calculations with a coupled strongly implicit method. Part 1: Finite difference solutions. No. 79-0011, 17th Aerospace Sciences Meeting, New Orleans, LA, Jan. 15-17, 1979.

21. Sune B. Berndt and Hans Sorenson, Transonic flow at a slotted test section wall. Proceedings, 4lst Semiannual Meeting of the Supersonic Tunnel Association, Los Angeles, CA, Mar. 28-29, 1974. 


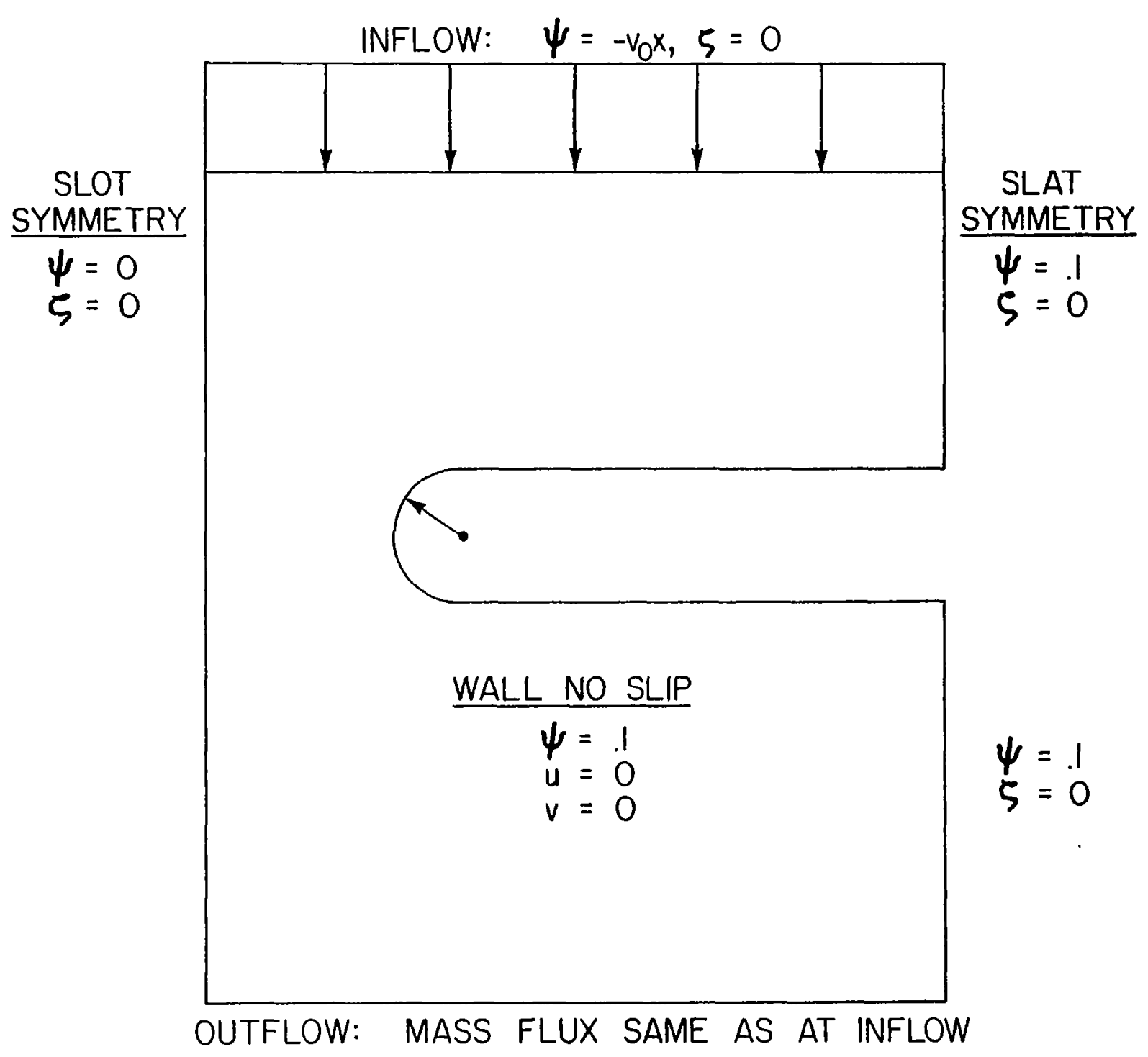




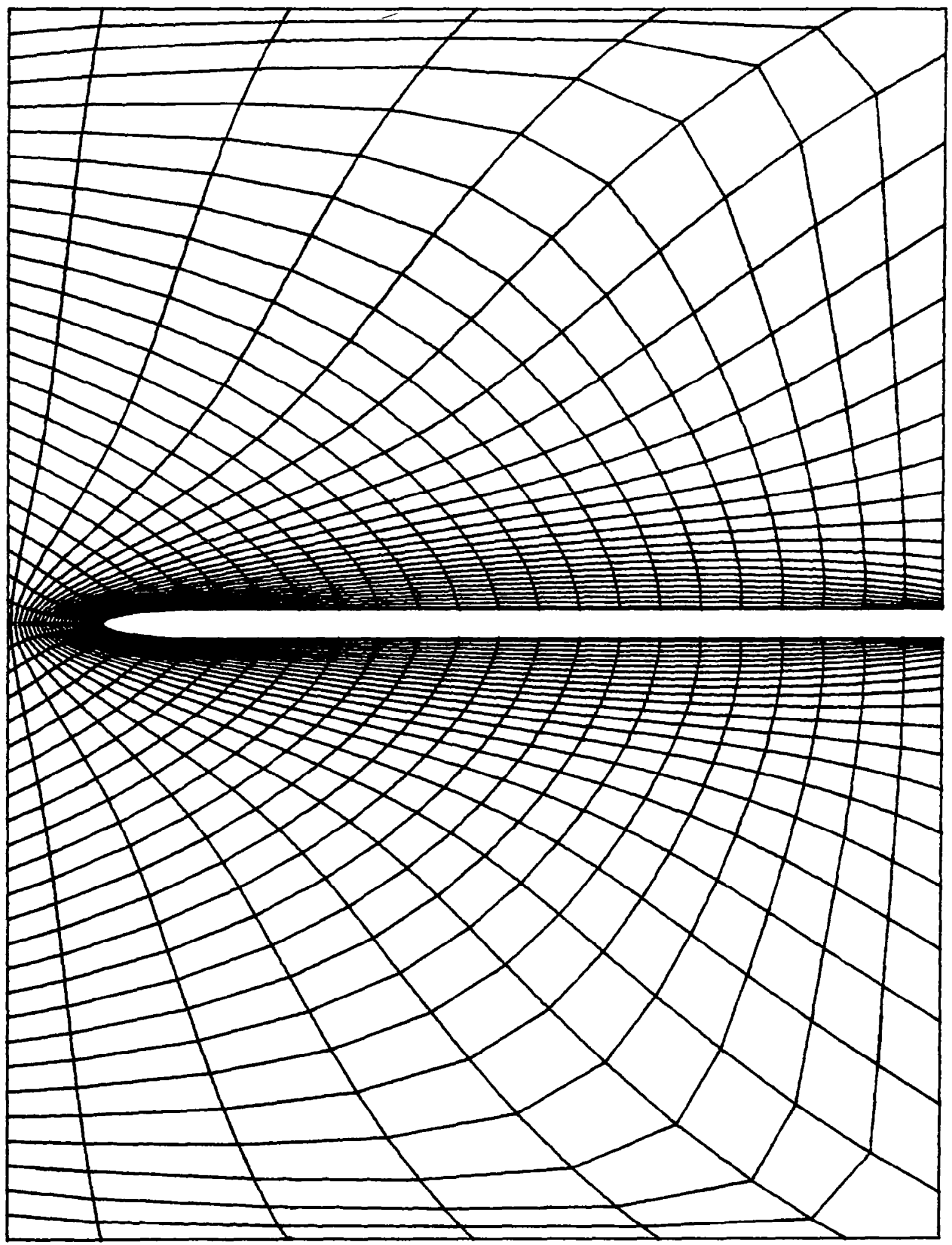

Figure $1(b)$. Coordinate system (alternate lines parallel to wall omitted) $6 \times 1$ magnified, horizontal $x$-direction. 


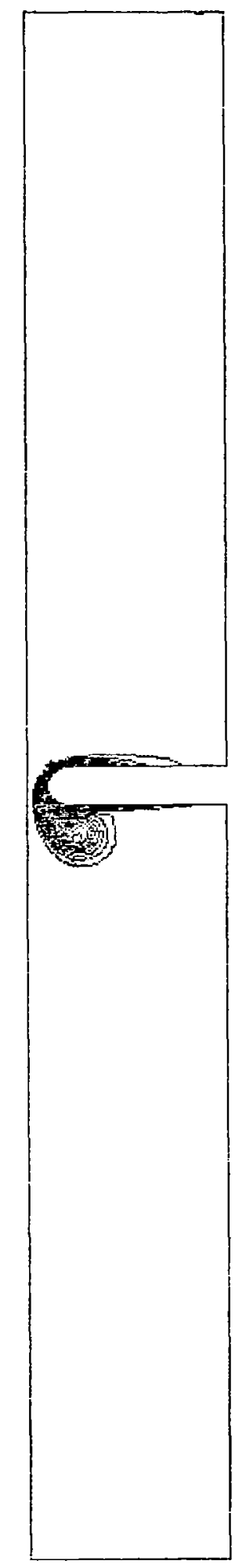

(a) VORTICITY $\operatorname{Re}=100$

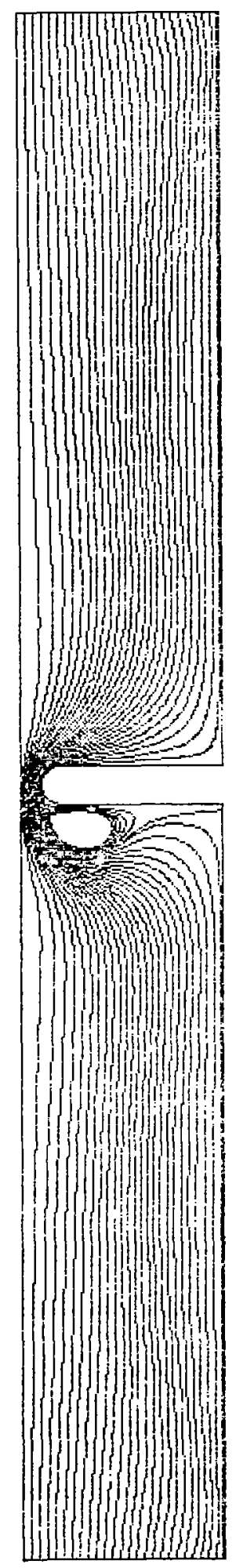

(b) STREAM FUNCTION

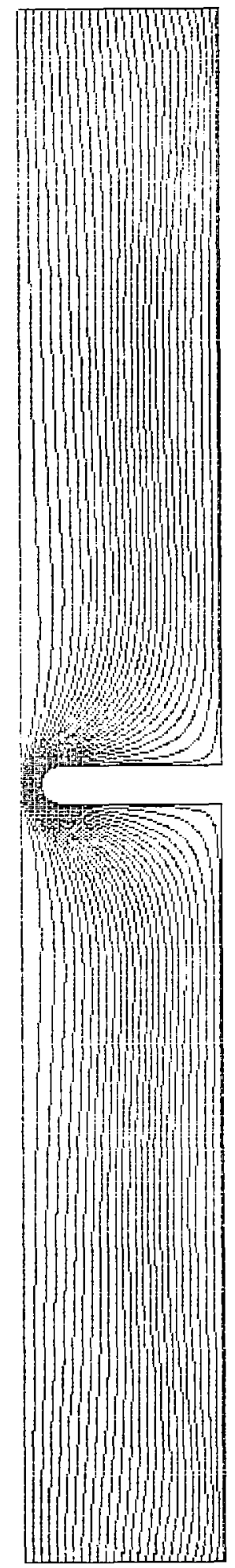

(c) INVISCID
STREAM
FUNCTION

Figure 2. Slot flow profiles: viscous vs. inviscid (continued). 

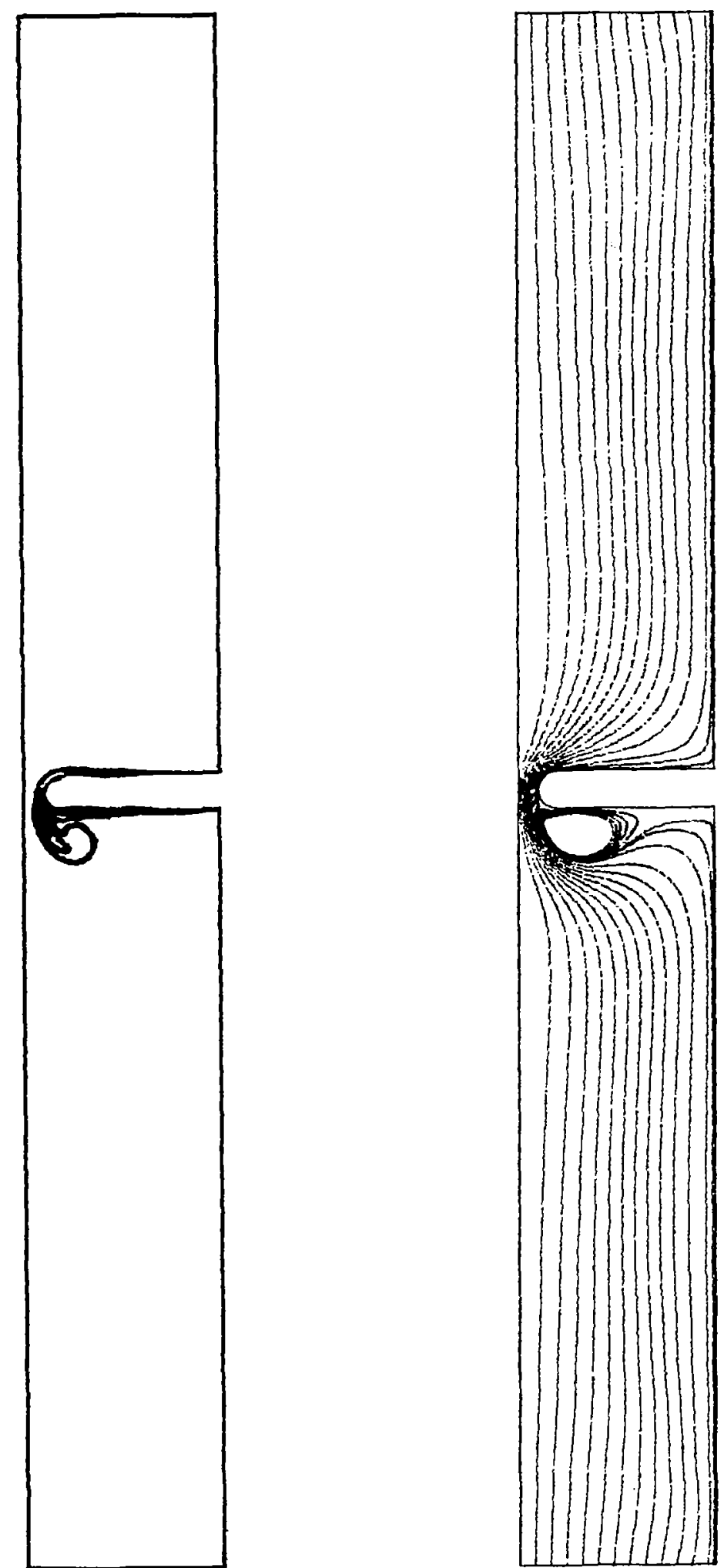

(d) VORTICITY (e) STREAM FUNCTION $\operatorname{Re}=1000$

Figure 2. Slot flow profiles: viscous vs, inviscid (concluded). 


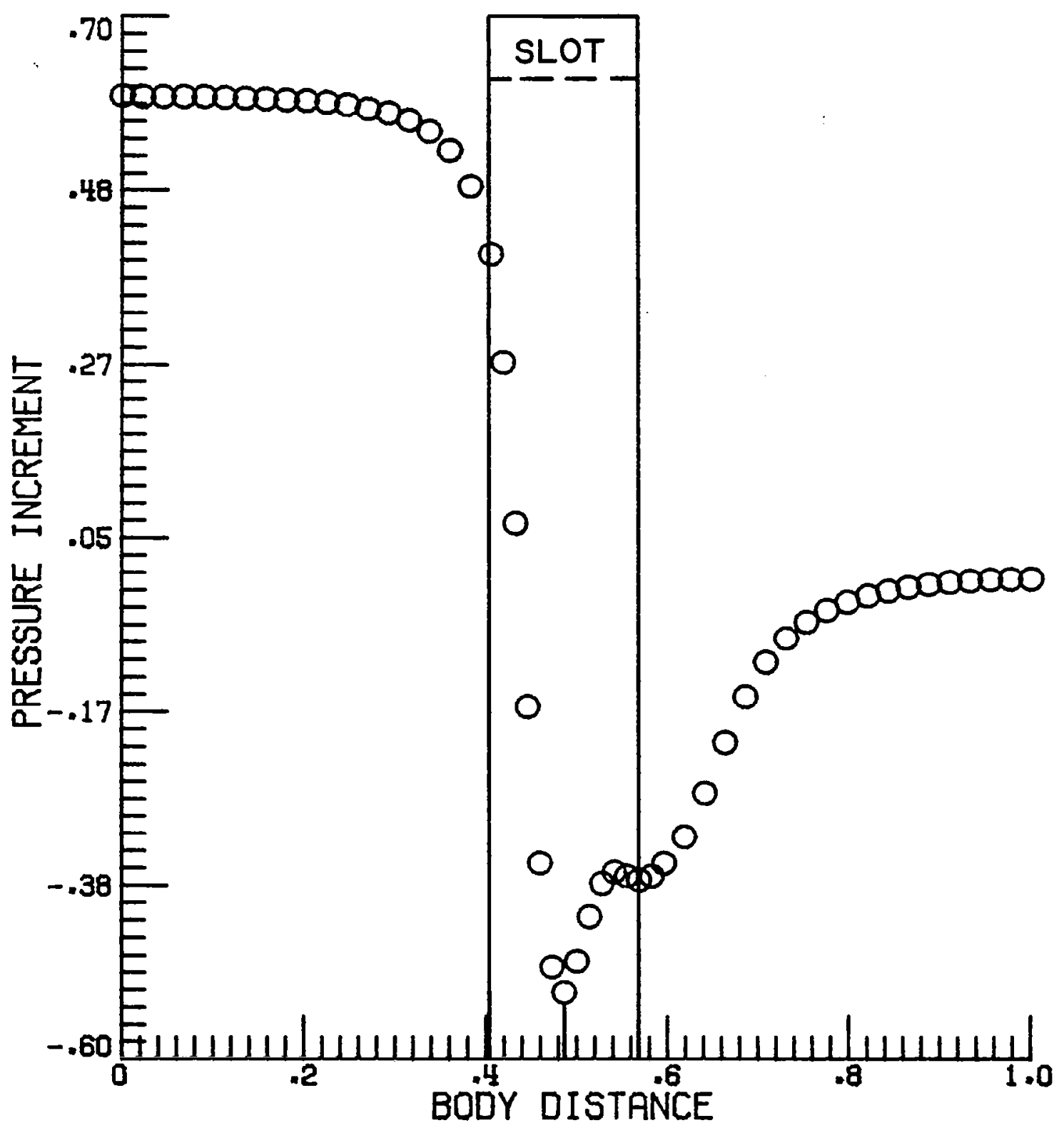

Figure 3. Body pressure distribution; $R e=100$, openness ratio $=0.1$. 


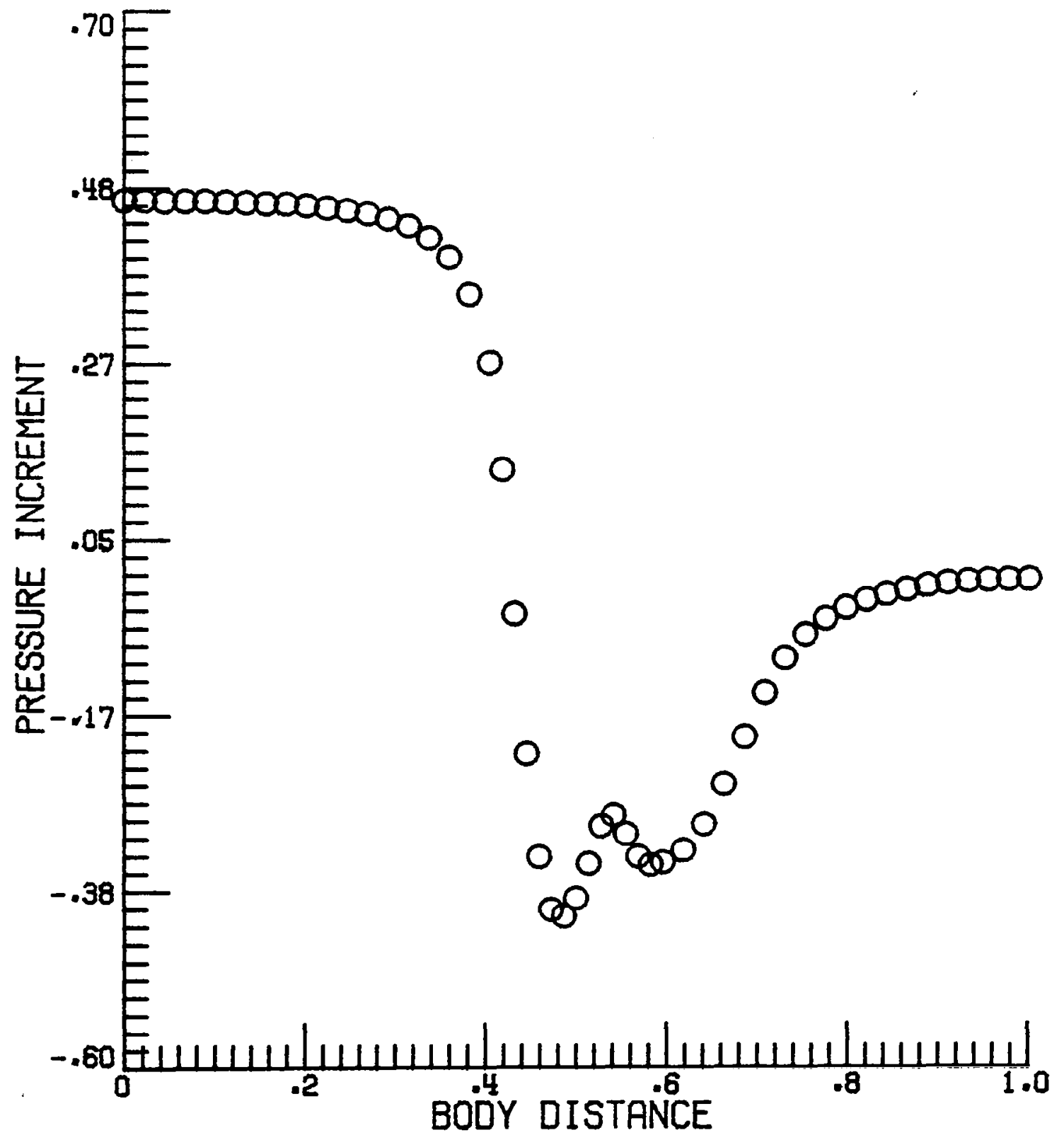

Figure 4. Body pressure distribution; $R e=1000$, openness ratio $=0.1$. 


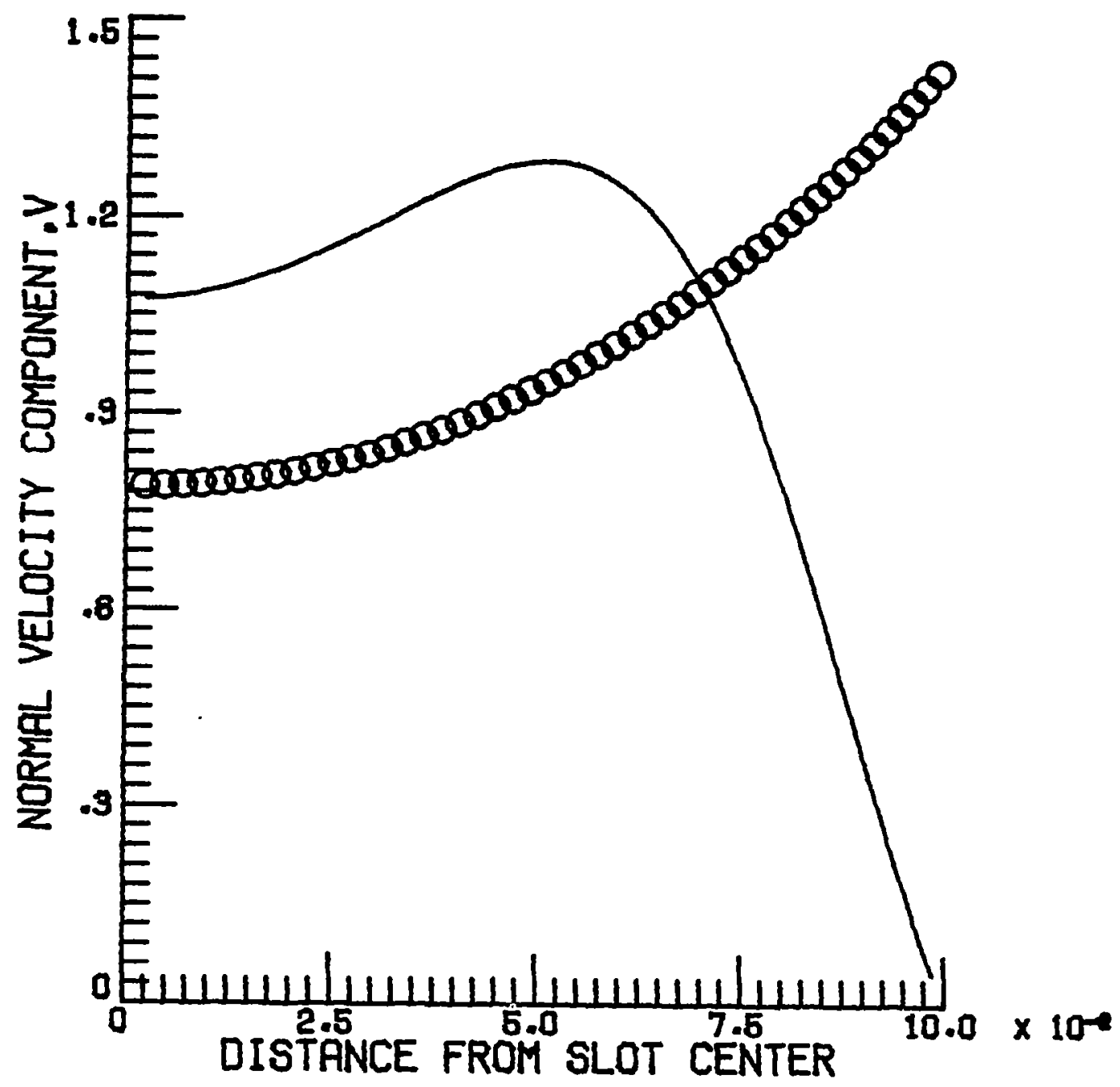

Figure 5. Slot normal velocity component at slat center: viscous $(\operatorname{Re}=100)$ vs. inviscid flow. 


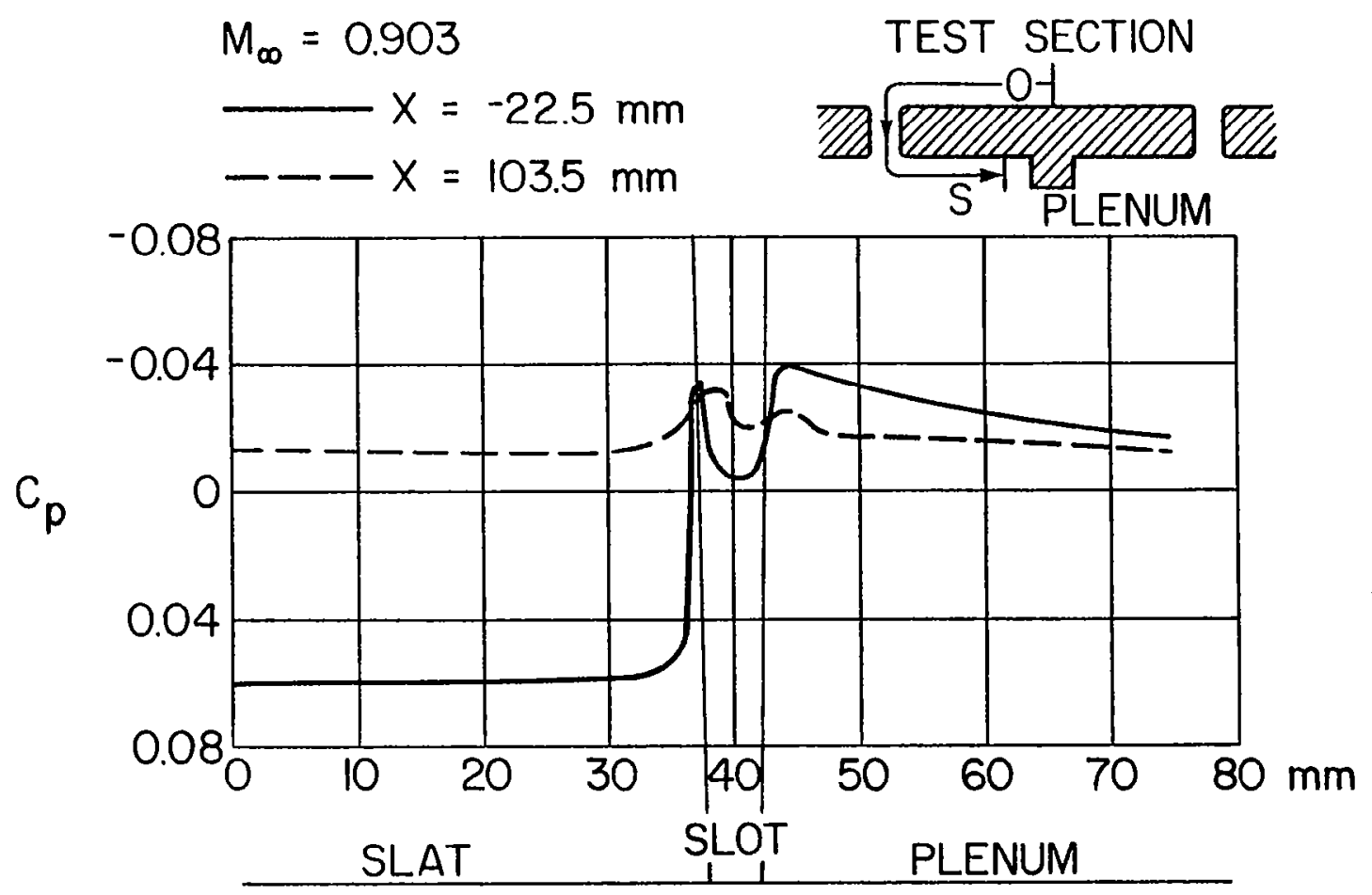

Figure 6. Experimental pressure distribution through slot under two conditions of flow [21]. 


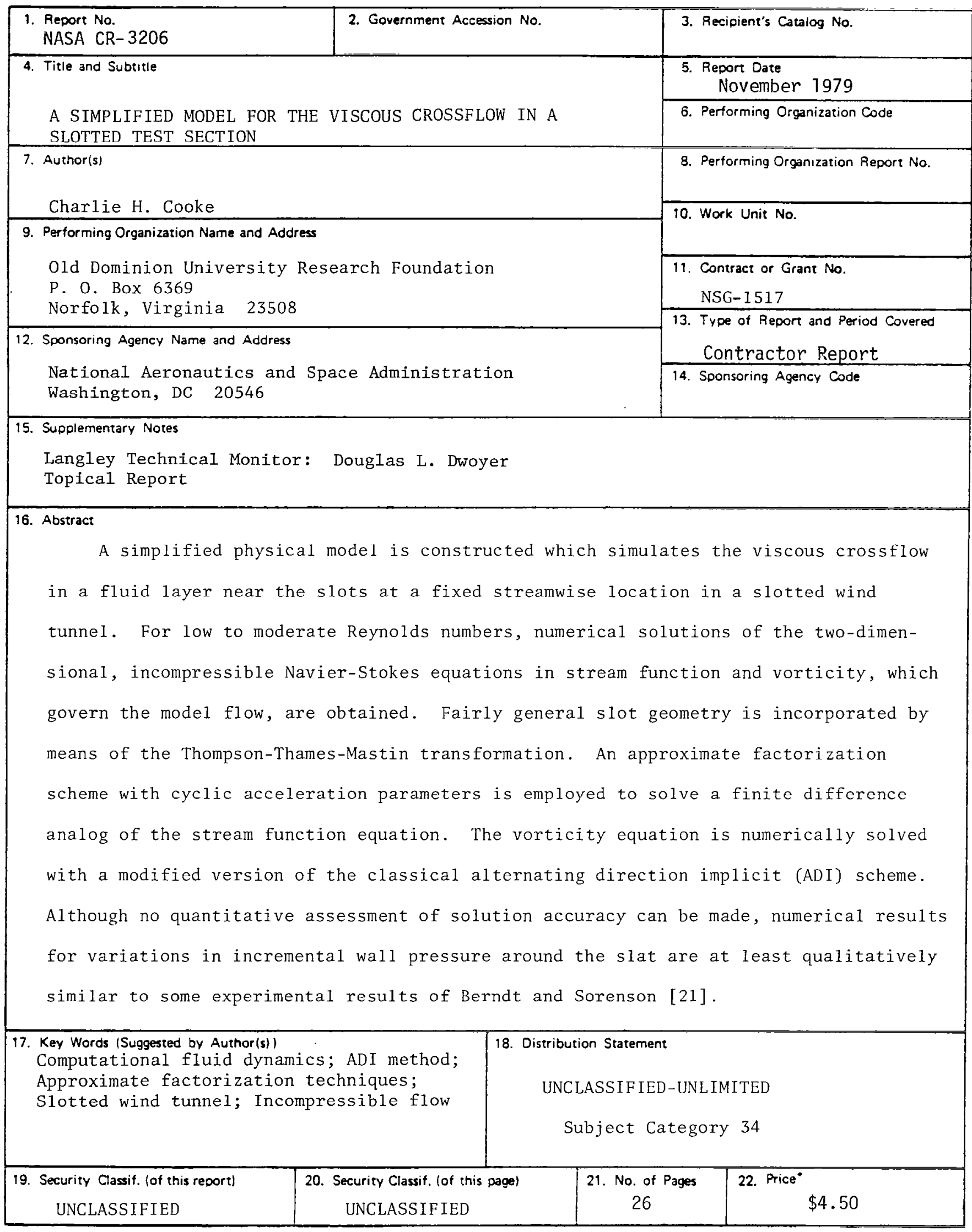

